\title{
DESIGN OF THE VIBRATING BARRIER DEVICE FOR A CLUSTER OF BUILDING UNDER STOCHASTIC EXCITATION
}

\author{
J. D. CORONADO JIMENEZ, A.TOMBARI AND P. CACCIOLA
}

School of Environment and Technology, University of Brighton, Cockcroft Building Lewes Road BN2 4GJ, Brighton, UK.

\begin{abstract}
The design of the novel Vibrating Barrier (ViBa) passive control device for the control of a cluster of buildings under Gaussian ground motion process is investigated in this paper. A simplified discrete lumped parameter model is used to represent the cluster of the buildings. The ViBa is modelled as a 2-DoF system able to interact through Winkler-type elastic springs with all the structures in the cluster. The structural parameters of the device are determined by minimizing in a least square sense the selected penalty function comprising the second order statistical moments of the relative displacements of the structures. Due to the different properties of the structures to be controlled the optimization procedure converges toward the minimization of the structure with higher energy. Various weighing have been considered to shift the control from a structure to another and to find the optimal solution so to guarantee a significant reduction of the response in the cluster avoiding any detrimental effects in any structure. Pertinent Monte Carlo study has been conducted to validate the optimization procedure in terms of mean values of the peak responses. Results from the Monte Carlo study confirm the accuracy of the optimization procedure presented in this paper.
\end{abstract}

Keywords: Vibrating Barrier, ViBa, Vibration Control, Cluster of Buildings

\section{Introduction}

Recent disasters in Mexico, Ecuador, Italy and Japan manifest the clear need to address the seismic resilience of existing buildings in a different and hopefully more affordable way. Structures are very seldom found in complete isolation, in fact the opposite seems to be the most common case. Buildings are often surrounded by adjacent structures in most scenarios, particularly in densely populated urban environments. While the effect of the specific site conditions is widely known, recent research (Schwan et al. 2016; Kham et al., 2013; Semblat et al., 2008; Gueguen et al., 2002) has also indicated the possible modification of the ground motion due to the presence of the built environment. In light of new research and better understanding of the complex phenomena of multiple interactions that take place in urban environments, innovative solutions have been proposed in the literature. One possible strategy is to protect the structures through trenches or sheet-pile walls in the soil (see e.g. Woods RD, 1968) for altering the displacement field based on the reflection, scattering and diffraction of dynamic surface waves or through the use of most innovative metamaterials, as the resonant metawedge (Colombi et al., 2016) for controlling the flow of Rayleigh waves. However, this approach is more effective for surface waves rather than body waves as those mainly produced by an earthquake. Cacciola and Tombari (2015) introduced for the first time, a non-localized solution, called Vibrating Barrier (ViBa), hosted in the soil and detached from the structures. The ViBa exploits the structure-soil-structure mechanism as a means of reducing the vibrations of structures due to seismic excitation or ground motion action. Analyses on the efficiency of the ViBa in protecting a single building are reported in Cacciola and Tombari (2015), in Cacciola et al. (2015) for structure founded on monopile foundation, in Tombari et al. (2016) for an industrial building and in Tombari et al. (2018) for cluster of buildings under deterministic excitation. The Vibrating Barrier interacts with the adjacent structures and mitigates their vibrations if opportunely designed. The tuning of the ViBa is addressed by resorting to a discrete model as already done in the literature where several authors have obtained rigorous analytical formulations from discrete SSSI problems. Kobori et al. (1973) defined a multi-spring-mass system for investigating the dynamic coupling of two adjacent square superficial foundations. Mulliken and Karabalis (1998) defined a simple discrete model for predicting the dynamic interaction between adjacent rigid surface foundations supported by a homogeneous, isotropic and linear elastic half-space. Recently, Alexander et al. (2013) developed a discrete model to study the SSSI problem of surface foundations by considering stochastic ground motion excitation which has been extended by Aldaikh et al. (2015 and 2016) to the case of three buildings and validated by means of experimentally shake-table testing. 
The design of the ViBa for a cluster of buildings in urban environments under ground motion stochastic excitation is explored in this paper. The buildings are modelled as 2-DOF systems coupled with the soil and with the adjacent buildings through linear elastic springs representing the soil-structure interactions and the structure-soil-structure interactions mechanism respectively. The ViBa device is modelled as a vibrating structure embedded in a foundation buried in the soil. The basic design principle is based on the minimization of a penalty function encompassing the stationary second order statistics of the structural response related to the peak response. Numerical results show the potentially of the vibrating barrier to successfully control the whole cluster.

\section{Non-localised vibration control in urban environments: Problem position}

Consider the global system depicted in Figure 1 under ground motion excitation at the bedrock $\ddot{\mathrm{u}}_{\mathrm{g}}(\mathrm{t})$. The Vibrating Barrier $(\mathrm{ViBa})$ is included aiming to reduce the vibration of the surrounding buildings.

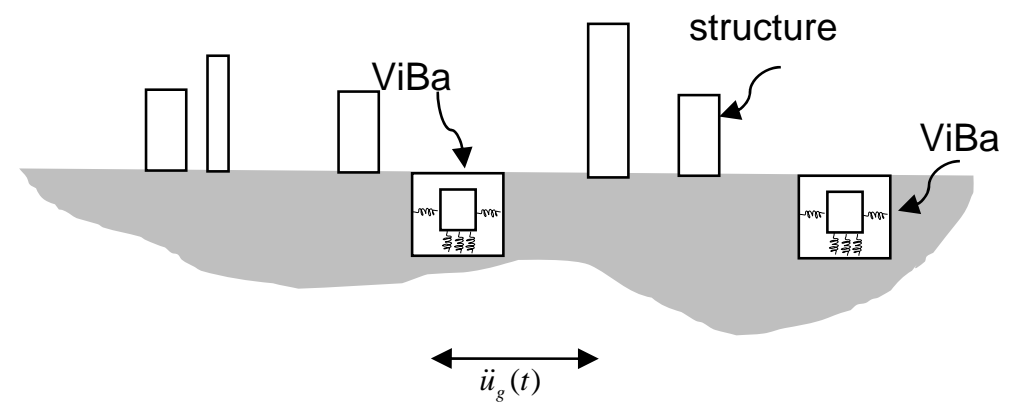

Figure 1 Sketch of the simplified model of structure in urban environment protected by the ViBa.

The equations governing the dynamic behaviour of the coupled system are derived in terms of absolute displacements, as it is conventional in soil-structure interaction, namely the dynamics of the problem take the form:

$$
\mathbf{M}(\boldsymbol{\alpha}) \ddot{\mathbf{u}}(\boldsymbol{\alpha}, \mathrm{t})+\mathbf{C}(\boldsymbol{\alpha}) \dot{\mathbf{u}}(\boldsymbol{\alpha}, \mathrm{t})+\mathbf{K}(\boldsymbol{\alpha}) \mathbf{u}(\boldsymbol{\alpha}, \mathrm{t})=\mathbf{Q}_{\mathrm{e}}(\boldsymbol{\alpha}) \mathrm{u}_{\mathrm{g}}(\mathrm{t})+\mathbf{Q}_{\mathrm{d}}(\boldsymbol{\alpha}) \dot{\mathrm{u}}_{\mathrm{g}}(\mathrm{t})
$$

where $\mathbf{M}(\boldsymbol{\alpha}), \mathbf{C}(\boldsymbol{\alpha})$ and $\mathbf{K}(\boldsymbol{\alpha})$ are the global mass, viscous damping and stiffness matrix, respectively; $\ddot{\mathbf{u}}(\boldsymbol{\alpha}, \mathrm{t}), \dot{\mathbf{u}}(\boldsymbol{\alpha}, \mathrm{t})$ and $\mathbf{u}(\boldsymbol{\alpha}, \mathrm{t})$ are respectively the absolute acceleration, velocity and displacement vector. Note that the ViBa dynamic behaviour is embedded in the global equation of motion through its structural parameters listed in the vector $\boldsymbol{\alpha}$ that represent the unknowns of the problem. The matrices of the global system are partitioned in the sub-matrices defined for the individual buildings and the ViBas; therefore the global mass matrix is as follows:

$$
\mathbf{M}(\boldsymbol{\alpha})=\left[\begin{array}{ccccc}
\mathbf{M}_{1} & 0 & & 0 & 0 \\
0 & \mathbf{M}_{\mathbf{i}} & \cdots & 0 & 0 \\
\multicolumn{2}{c}{} & & \ddots & \multicolumn{2}{c}{\vdots} \\
0 & 0 & & \mathbf{M}_{\mathbf{n}} & 0 \\
0 & 0 & & 0 & \mathbf{M}_{\mathbf{v}}
\end{array}\right]
$$

in which the ith sub-block includes the mass of the ith structure, while $\mathbf{M}_{\mathbf{v}}$ is the mass matrix of the ViBas distributed in the urban environment given by 


$$
\mathbf{M}_{\mathbf{v}}=\left[\begin{array}{ccccc}
\mathbf{M}_{\mathbf{v} 1} & 0 & \ldots & 0 & 0 \\
0 & \mathbf{M}_{\mathbf{v} 2} & & 0 & 0 \\
\multicolumn{2}{c}{} & & \ddots & \multicolumn{2}{c}{\vdots} \\
0 & 0 & & \mathbf{M}_{\mathbf{v i}} & 0 \\
0 & 0 & & 0 & \mathbf{M}_{\mathbf{v n}}
\end{array}\right]
$$

With

$$
\mathbf{M}_{\mathrm{Vi}}=\left[\begin{array}{cc}
\mathbf{m}_{\mathrm{ViBa}, \mathrm{i}} & 0 \\
0 & \mathbf{m}_{\mathrm{f}, \mathrm{ViBa}, \mathrm{i}}
\end{array}\right]
$$

The global damping matrix $\mathbf{C}(\boldsymbol{\alpha})$ and the global stiffness matrix $\mathbf{K}(\boldsymbol{\alpha})$ are block-matrices partitioned in the following form:

$$
\mathbf{C}(\boldsymbol{\alpha})=\left[\begin{array}{cccccc}
\mathbf{C}_{1} & \mathbf{C}_{1, \mathbf{i}} & \ldots & \mathbf{C}_{1, \mathbf{n}} & \mathbf{C}_{1, \mathbf{v}} \\
\mathbf{C}_{\mathbf{i}, 1} & \mathbf{C}_{i} & & \mathbf{C}_{\mathbf{i}, \mathbf{n}} & \mathbf{C}_{\mathbf{i}, \mathbf{v}} \\
& \vdots & \ddots & & \vdots \\
\mathbf{C}_{\mathbf{n}, 1} & \mathbf{C}_{\mathbf{n}, \mathbf{i}} & \ldots & \mathbf{C}_{\mathbf{n}} & \mathbf{C}_{\mathbf{n}, \mathbf{v}} \\
\mathbf{C}_{\mathbf{v}, 1} & \mathbf{C}_{\mathbf{v}, \mathbf{i}} & & \mathbf{C}_{\mathbf{v}, \mathbf{n}} & \mathbf{C}_{\mathbf{v}}
\end{array}\right]
$$

for the damping matrix, while:

$$
\mathbf{K}(\boldsymbol{\alpha})=\left[\begin{array}{cccccc}
\mathbf{K}_{1} & \mathbf{K}_{1, \mathbf{i}} & \ldots & \mathbf{K}_{1, \mathbf{n}} & \mathbf{K}_{1, \mathbf{V}} \\
\mathbf{K}_{\mathbf{i}, 1} & \mathbf{K}_{\mathbf{i}} & & \mathbf{K}_{\mathbf{i}, \mathbf{n}} & \mathbf{K}_{\mathbf{i}, \mathbf{V}} \\
& \vdots & & \ddots & & \vdots \\
\mathbf{K}_{\mathbf{n}, 1} & \mathbf{K}_{\mathbf{n}, \mathbf{i}} & \ldots & \mathbf{K}_{\mathbf{n}} & \mathbf{K}_{\mathbf{n}, \mathbf{V}} \\
\mathbf{K}_{\mathbf{V}, 1} & \mathbf{K}_{\mathbf{V}, \mathbf{i}} & & \mathbf{K}_{\mathbf{V}, \mathbf{n}} & \mathbf{K}_{\mathbf{V}}
\end{array}\right]
$$

is the stiffness matrix. The main diagonal sub-matrices $\mathbf{C}_{i}$ and $\mathbf{K}_{i}(\mathrm{r}=1, \ldots, \mathrm{n})$ describe the viscous damping and stiffness matrix of the rth-structure and its interaction with the soil. The matrices $\mathbf{C}_{\mathbf{v}}$ and $\mathbf{K}_{\mathbf{v}}$ define the damping and stiffness matrix of the $\mathrm{ViBa}$ and its interactions to the other buildings through the soil. Lastly, the off-diagonal sub-matrices $\mathbf{C}_{i j}$ and $\mathbf{K}_{i j}(\mathrm{i}, \mathrm{j}=1, \ldots, \mathrm{n})$ are related to the dynamic coupling between the ith and the jth structures. By considering the state space

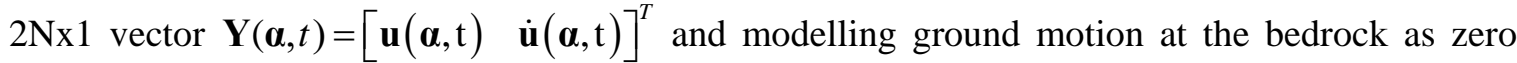
mean Gaussian stationary white noise with power spectral density $S_{W}$, the evolution of the second order moments in the nodal space is ruled by the following ordinary $2 \mathrm{Nx} 2 \mathrm{~N}$ set differential equation

$$
\dot{\mathbf{m}}_{\mathbf{Y}}{ }^{(2)}(\boldsymbol{\alpha}, t)=\mathbf{D}_{2}(\boldsymbol{\alpha}) \mathbf{m}_{\mathbf{Y}}{ }^{(2)}(\boldsymbol{\alpha}, t)+2 \pi S_{W} \overline{\mathbf{V}}^{[2]}(\boldsymbol{\alpha})
$$

Where

$$
\begin{aligned}
& \mathbf{m}_{\mathbf{Y}}{ }^{(2)}(\boldsymbol{\alpha}, t)=E[\mathbf{Y}(\boldsymbol{\alpha}, t) \otimes \mathbf{Y}(\boldsymbol{\alpha}, t)] \\
& \mathbf{D}_{2}(\boldsymbol{\alpha})=\mathbf{D}(\boldsymbol{\alpha}) \otimes \mathbf{I}_{2 N}+\mathbf{I}_{2 N} \otimes \mathbf{D}(\boldsymbol{\alpha})
\end{aligned}
$$

Where $\mathbf{I}_{2 N}$ is the identity matrix of order $2 \mathrm{Nx} 2 \mathrm{~N}$ and 


$$
\overline{\mathbf{V}}^{[2]}(\boldsymbol{\alpha})=\mathbf{V}(\boldsymbol{\alpha}) \otimes \mathbf{V}(\boldsymbol{\alpha})
$$

Also in Equations (8)-(10) $\otimes$ stands for Kronecker product.

\section{Optimization procedure}

In the previous formulation, the structural parameters of the ViBa indicated by the generic vector $\boldsymbol{\alpha}$ represent the unknowns of the problem to be determined. Therefore, various optimization criteria can be used to determine the unknown parameters. In this paper the optimization is defined by the minimization of the penalty function $\mathbf{J}(\boldsymbol{\alpha})$ in a lest square sense, that is

$$
\begin{aligned}
& \min \{\mathbf{J}(\boldsymbol{\alpha})\} \\
& \boldsymbol{\alpha}=\left\{\mathbf{K}_{\mathbf{v}}, \mathbf{M}_{\mathbf{v}}, \mathbf{C}_{\mathbf{v}}\right\} \in \mathbb{R}_{0}^{+}
\end{aligned}
$$

where $\mathbf{J}(\boldsymbol{\alpha})$ is defined in terms of either relative displacements, internal forces, energy etc. In this regard, consider the Taylor series expansion of a generic response parameter $\boldsymbol{x}(\boldsymbol{\alpha})$ truncated at the first order (Friswell and Mottershead, 1995),

$$
x(\alpha)=x\left(\alpha_{o}\right)+S_{\alpha_{o}}\left(\alpha-\alpha_{o}\right)+\ldots
$$

where $\boldsymbol{S}_{a_{o}}$ is the sensitivity matrix, of order r x p, containing the first partial derivative of the response $\boldsymbol{x}(\boldsymbol{\alpha})$ with respect to the unknown parameters $\boldsymbol{\alpha}$ determined for an initial set of parameters $\boldsymbol{\alpha}_{o}$ :

$$
S_{a_{o}}=\left[\frac{\partial}{\partial \boldsymbol{\alpha}_{1}} \boldsymbol{x}(\boldsymbol{\alpha}) \cdots \frac{\partial}{\partial \boldsymbol{\alpha}_{p}} \boldsymbol{x}(\boldsymbol{\alpha})\right]
$$

If the Taylor series is restricted to the first two terms the following linear approximation can be cast.

$$
\delta \boldsymbol{x}=\boldsymbol{S}_{a_{o}} \delta \alpha
$$

Where $\boldsymbol{\delta} \boldsymbol{x}=\boldsymbol{x}(\boldsymbol{\alpha})-\boldsymbol{x}\left(\boldsymbol{\alpha}_{\boldsymbol{o}}\right)$ is the error in the output and $\boldsymbol{\delta} \boldsymbol{\alpha}=\boldsymbol{\alpha}-\boldsymbol{\alpha}_{o}$ is the change in the parameters. Equation (14) represents a set of linear equations with the unknown parameters of vector $\boldsymbol{\delta} \boldsymbol{\alpha}$, if the sensitivity matrix is square and invertible the solution is trivial. However, if the sensitivity matrix is rectangular the system could be either over-determined $(r>p)$ or under-determined $(r<p)$. In which case the problem can be solved by the usage of the Moore-Penrose pseudo inverse procedure as in Friswell and Mottershead (1995) and presented below

$$
\boldsymbol{\delta} \boldsymbol{\alpha}=\left[\boldsymbol{S}_{\alpha_{o}}{ }^{T} \boldsymbol{S}_{\boldsymbol{\alpha}_{o}}\right]^{-1} \boldsymbol{S}_{\alpha_{o}}{ }^{T} \boldsymbol{\delta} \boldsymbol{x}
$$

Equation (15) can be casted in a form that would suit an iterative procedure as follows

$$
\boldsymbol{\alpha}_{i+1}=\boldsymbol{\alpha}_{i}+\left[\boldsymbol{S}_{a_{i}}{ }^{T} \boldsymbol{S}_{a_{i}}\right]^{-1} \boldsymbol{S}_{a_{i}}{ }^{T}\left(\boldsymbol{x}_{T}-\boldsymbol{x}_{i}\right)
$$

Where $\boldsymbol{\alpha}_{i}$ represent the vector of unknown parameters at the iteration $i$ and $\boldsymbol{x}_{T}-\boldsymbol{x}_{i}$ is the difference between the target response $\boldsymbol{x}_{T}$ and that estimated $\boldsymbol{x}_{i}$ for the parameter set $\boldsymbol{\alpha}_{i}$. Equation (16) 
represents the iterative solution of equation (11). It is important to mention that any parameter that has an influence in the model response can be included in the unknowns parameter vector $\boldsymbol{\alpha}_{i}$. However, two important aspects need to be considered, namely, the parameters must be in the same order of magnitude for the problem not to become ill-conditioned otherwise scaling factors should be introduced to improve the numerical conditioning of the problem. Secondly, the procedure is based in the assumption that equation (14) represents a combination of independent linear equations, if a parameter has none or very little influence in the measured model response vector $\boldsymbol{x}_{i}$, the problem becomes illconditioned and additional measures need to be taken (see Friswell and Mottershead, 1995). In this paper the vector $\mathbf{x}$ is defined in terms of the second order stationary response statistical moments, that is

$$
\mathbf{x}=\mathbf{P} \mathbf{m}_{\mathbf{Y}}{ }^{(2)}(\boldsymbol{\alpha})
$$

Where $\mathbf{P}$ is a matrix whose elements are either null or unitary so to extract from the vector $\mathbf{m}_{\mathbf{Y}}{ }^{(2)}(\boldsymbol{\alpha})$ the relevant statistical quantities. The stationary response of the second order statistics is given by the following equation

$$
\mathbf{m}_{\mathbf{Y}}{ }^{(2)}(\boldsymbol{\alpha}, t)=\mathbf{m}_{\mathbf{Y}}{ }^{(2)}(\boldsymbol{\alpha})=-\mathbf{D}_{2}{ }^{-1}(\boldsymbol{\alpha}) 2 \pi S_{W} \overline{\mathbf{V}}^{[2]}(\boldsymbol{\alpha})
$$

The sensitivity of the stochastic response respect to the vector of the parameters $\boldsymbol{\alpha}$ is determined (see e.g. Cacciola et al 2005; Cacciola et al 2011) as follows:

$$
\boldsymbol{S}_{Y}^{(2)}(\boldsymbol{\alpha}, t)=\frac{\partial \boldsymbol{m}_{\boldsymbol{Y}}{ }^{(2)}(\boldsymbol{\alpha}, t)}{\partial \boldsymbol{\alpha}}=E\left[\boldsymbol{S}_{Y}(\boldsymbol{\alpha}, t) \otimes \boldsymbol{Y}(\boldsymbol{\alpha}, t)\right]+E\left[\boldsymbol{Y}(\boldsymbol{\alpha}, t) \otimes \boldsymbol{S}_{Y}(\boldsymbol{\alpha}, t)\right]
$$

Therefore, the evolution of the sensitivity can be written in the form

$$
\dot{\boldsymbol{S}}_{Y}{ }^{(2)}(\boldsymbol{\alpha}, t)=\boldsymbol{A}_{2}(\boldsymbol{\alpha}) \boldsymbol{m}_{\boldsymbol{Y}}{ }^{(2)}(\boldsymbol{\alpha}, t)+\boldsymbol{D}_{2}(\boldsymbol{\alpha}) \boldsymbol{S}_{Y}{ }^{(2)}(\boldsymbol{\alpha}, t)+\boldsymbol{B}_{2}(\boldsymbol{\alpha}) 2 \pi S_{W}
$$

With

$$
\begin{aligned}
& A_{2}(\boldsymbol{\alpha})=A(\boldsymbol{\alpha}) \otimes \boldsymbol{I}_{2 N}+I_{2 N} \otimes A(\boldsymbol{\alpha}) \\
& \mathbf{B}_{2}(\boldsymbol{\alpha})=\mathbf{B}(\boldsymbol{\alpha}) \otimes \overline{\mathbf{V}}(\boldsymbol{\alpha})+\overline{\mathbf{V}}(\boldsymbol{\alpha}) \otimes \mathbf{B}(\boldsymbol{\alpha})
\end{aligned}
$$

Where $\boldsymbol{A}(\boldsymbol{\alpha})$ and $\mathbf{B}(\boldsymbol{\alpha})$ are defined as

$$
\boldsymbol{A}(\boldsymbol{\alpha})=\frac{\partial \boldsymbol{D}(\boldsymbol{\alpha})}{\partial \boldsymbol{\alpha}} ; \quad \boldsymbol{B}(\boldsymbol{\alpha})=\frac{\partial \boldsymbol{V}(\boldsymbol{\alpha})}{\partial \boldsymbol{\alpha}}
$$

The stationary solution of equation (20) can be then written in the following form

$$
\boldsymbol{S}_{Y}{ }^{(2)}(\boldsymbol{\alpha})=-\boldsymbol{D}_{2}^{-1}(\boldsymbol{\alpha})\left[\boldsymbol{A}_{2}(\boldsymbol{\alpha}) \boldsymbol{m}_{\boldsymbol{Y}}{ }^{(2)}(\boldsymbol{\alpha}, t)+\boldsymbol{B}_{2}(\boldsymbol{\alpha}) 2 \pi S_{W}\right]
$$

Therefore,

$$
\mathbf{S}_{\boldsymbol{\alpha}_{i}}=\left.\mathbf{P S}_{Y}^{(2)}(\boldsymbol{\alpha})\right|_{\boldsymbol{\alpha}=\boldsymbol{a}_{i}}
$$

In order to minimize the peak response of the selected cluster the target response vector $\mathbf{x}_{T}$ is herein selected as a null vector aiming to converge to an optimal solution where most of the input energy 
is absorbed by the ViBa so to protect the whole cluster. Pertinent boundaries can be also selected to the solution to avoid unrealistic results or detrimental effect is some of the buildings.

\section{Numerical results}

Consider the discrete model represented in Figure 2. It represents a simplified model of a cluster of buildings extracted by an urban environment and the ViBa. The discrete parameters pertinent to the buildings can be calibrated through an identification procedure, so are considered know quantities.

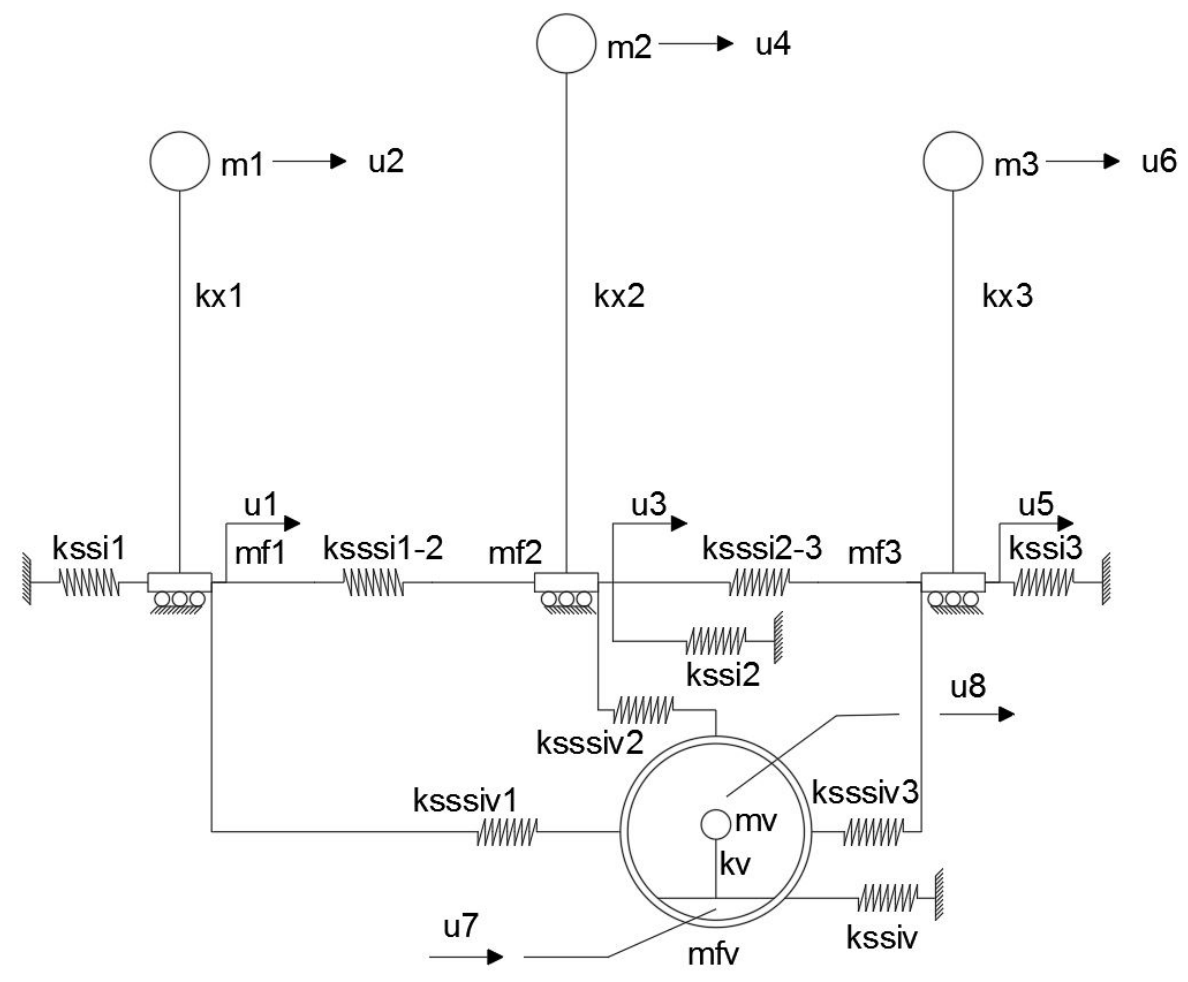

Figure 2 Discrete model of a cluster of buildings coupled with a SDOF ViBa.

The dynamic response of this system is governed by equation (1) where the mass and stiffness matrix are the following:

$$
\mathbf{M}=\left[\begin{array}{cccccccc}
m_{1} & 0 & 0 & 0 & 0 & 0 & 0 & 0 \\
0 & m_{f 1} & 0 & 0 & 0 & 0 & 0 & 0 \\
0 & 0 & m_{2} & 0 & 0 & 0 & 0 & 0 \\
0 & 0 & 0 & m_{f 2} & 0 & 0 & 0 & 0 \\
0 & 0 & 0 & 0 & m_{3} & 0 & 0 & 0 \\
0 & 0 & 0 & 0 & 0 & m_{f 3} & 0 & 0 \\
0 & 0 & 0 & 0 & 0 & 0 & m_{f v} & 0 \\
0 & 0 & 0 & 0 & 0 & 0 & 0 & m_{v}
\end{array}\right]
$$




$$
\mathbf{K}=\left[\begin{array}{cccccccc}
k_{x 1} & -k_{x 1} & 0 & 0 & 0 & 0 & 0 & 0 \\
-k_{x 1} & k_{c 1} & 0 & -k_{s s s i 2} & 0 & 0 & -k_{s s s i v 1} & 0 \\
0 & 0 & k_{x 2} & -k_{x 2} & 0 & 0 & 0 & 0 \\
0 & -k_{s s s i 12} & -k_{x 2} & k_{c 2} & 0 & -k_{s s s i 23} & -k_{s s s i v 2} & 0 \\
0 & 0 & 0 & 0 & k_{x 3} & -k_{x 3} & 0 & 0 \\
0 & 0 & 0 & -k_{s s s i 23} & -k_{x 3} & k_{c 3} & -k_{s s s i v 3} & 0 \\
0 & -k_{s s s i v 1} & 0 & -k_{s s s i v 2} & 0 & -k_{s s i v 3} & k_{c 4} & -k_{v} \\
0 & 0 & 0 & 0 & 0 & 0 & -k_{v} & k_{v}
\end{array}\right]
$$

with

$$
\begin{aligned}
& k_{c 1}=k_{s s i 1}+k_{s s s i 12}+k_{s s s i v 1}+k_{x 1} \\
& k_{c 2}=k_{s s i 2}+k_{s s s i 2}+k_{s s s i 23}+k_{s s s i v 2}+k_{x 2} \\
& k_{c 3}=k_{s s i 3}+k_{s s s i 23}+k_{s s s i v 3}+k_{x 3} \\
& k_{c 4}=k_{s s i v}+k_{s s s i v 1}+k_{s s s i v 2}+k_{s s s i v 3}+k_{v}
\end{aligned}
$$

The damping matrix is calculated as a back transformation from the modal space assuming all the modes have the same identical damping $\zeta=0.05$. Tables 1-3 lists the mechanical parameter of the cluster of buildings used in the numerical application.

Table 1 Mass parameters of the lumped discrete model of cluster of buildings

\begin{tabular}{lllllll}
\hline Units/Parameter & $m_{1}$ & $m_{2}$ & $m_{3}$ & $m_{f 1}$ & $m_{f 2}$ & $m_{f 3}$ \\
\hline $\mathrm{kg}$ & $7.50 \mathrm{E} 4$ & $1.50 \mathrm{E} 5$ & $7.50 \mathrm{E} 4$ & $8.32 \mathrm{E} 5$ & $4.08 \mathrm{E} 5$ & $2.91 \mathrm{E} 6$ \\
\hline
\end{tabular}

Table 2 Stiffness parameters of the lumped discrete model of cluster of buildings coupled

\begin{tabular}{lllllll}
\hline Units/Parameter & $k_{x 1}$ & $k_{x 2}$ & $k_{x 3}$ & $k_{\text {ssi }} \mathrm{kss} 1$ & $k_{\text {ssi } 2}$ & $k_{\text {ssi3 }}$ \\
\hline $\mathrm{N} / \mathrm{m}$ & $7.68 \mathrm{E} 6$ & $5.90 \mathrm{E} 6$ & $1.02 \mathrm{E} 7$ & $6.61 \mathrm{E} 7$ & $3.70 \mathrm{E} 7$ & $1.15 \mathrm{E} 8$ \\
\hline
\end{tabular}

Table 3 Cross-Interaction Stiffness parameters of the lumped discrete model of cluster of buildings coupled

\begin{tabular}{lll}
\hline Units/Parameter & $k_{\text {ssi12 }}$ & $k_{\text {ssi23 }}$ \\
\hline $\mathrm{N} / \mathrm{m}$ & $2.05 \mathrm{E} 7$ & $6.64 \mathrm{E} 7$ \\
\hline
\end{tabular}

The objective of this numerical study is to apply the numerical procedure presented in section 2 to determine the optimal parameters of the ViBa so to minimize the stochastic response (in terms of second order statistical moments) of the three buildings. In this regard the selected elements of the response $3 \mathrm{x} 1$ vector $\mathbf{x}$ are the statistical moments of the relative displacements between the top and base of the structure. That is 


$$
\begin{aligned}
& x(1)=m_{11}{ }^{(2)}(\alpha)+m_{22}{ }^{(2)}(\alpha)-m_{12}{ }^{(2)}(\alpha)-m_{21}{ }^{(2)}(\alpha) \\
& x(2)=m_{33}{ }^{(2)}(\alpha)+m_{44}{ }^{(2)}(\alpha)-m_{34}{ }^{(2)}(\alpha)-m_{43}{ }^{(2)}(\alpha) \\
& x(3)=m_{55}{ }^{(2)}(\alpha)+m_{66}{ }^{(2)}(\alpha)-m_{56}{ }^{(2)}(\alpha)-m_{65}{ }^{(2)}(\alpha)
\end{aligned}
$$

In which $m_{i i}^{(2)}(\alpha)(i=1 \ldots 6)$ are the second order response statistical moments of the ith degree of freedom while $m_{j k}{ }^{(2)}(\alpha)(\mathrm{j}, \mathrm{k}=1 \ldots 6 j \neq k)$ are the cross statistical moments of the response between the jth and kth degrees of freedom.

Tables 4 and 5 list the mechanical parameters of the ViBa assigned a priori and not included in the optimization procedure.

Table 4 Mass parameters of the ViBa device

\begin{tabular}{lll}
\hline Units/Parameter & $m_{v}$ & $m_{f v}$ \\
\hline $\mathrm{kg}$ & $5.75 \mathrm{E} 5$ & $1.00 \mathrm{E} 3$ \\
\hline
\end{tabular}

Table 5 Soil-structure interaction and cross interaction stiffness parameters of the ViBa device

\begin{tabular}{lllll}
\hline Units/Parameter & $k_{\text {sssiv }}$ & $k_{\text {sssiv } 1}$ & $k_{\text {sssiv } 2}$ & $k_{\text {sssiv } 3}$ \\
\hline $\mathrm{N} / \mathrm{m}$ & $1.00 \mathrm{E} 7$ & $1.00 \mathrm{E} 7$ & $1.00 \mathrm{E} 7$ & $1.00 \mathrm{E} 7$ \\
\hline
\end{tabular}

Due to the simple configuration of the ViBa designed as an SDOF system the vector parameter $\boldsymbol{\alpha}$ therefore reduces to a scalar value representing the single unknown to be determined i.e. the stiffness parameter $k_{v}$. Considering the particular aspects of the response of the cluster it is observed the significant difference that exist between the amplitude of the displacements and the pertinent PSD of the central structure (structure 2) compared with the remaining two structures. This introduces additional constraints into the design process, since the unbounded minimisation of the response parameters will unavoidably aim to reduce the response of structure 2 rather than that of buildings 1 and 3. Scaling factors are also introduced in order to shift the solution towards a particular structure within the cluster. To illustrate this, four cases are analysed, namely, original case in which no scaling factors are applied, the case in which each structure is targeted individually. Figure present the results for the four scenarios considered. In the same figure the percentage difference between the areas of the PSD is also reported. As expected it is noticed that the solution of the minimisation problem with no modification factors results in significant reductions in the central structure (Figure ), nevertheless, the design yields results that are beneficial for all three structures. The solution when a scaling factor is applied to shift the solution towards protecting structure 1 are displayed in Figure, it is observed that a higher level of protection is achieved compared to the previous case, in addition reductions in building three are also noticed even though the solution is mainly intended for building one. This effects is due to the inability of the coupled ViBa system to achieve the frequency required to protect only building one, regardless of the increase in the stiffness of the resonant element within the ViBa the frequency of the coupled system does not increase. Further, detrimental effects occur in structure 2, where the power of the relative displacement in the presence of the $\mathrm{ViBa}$ is greater than the 

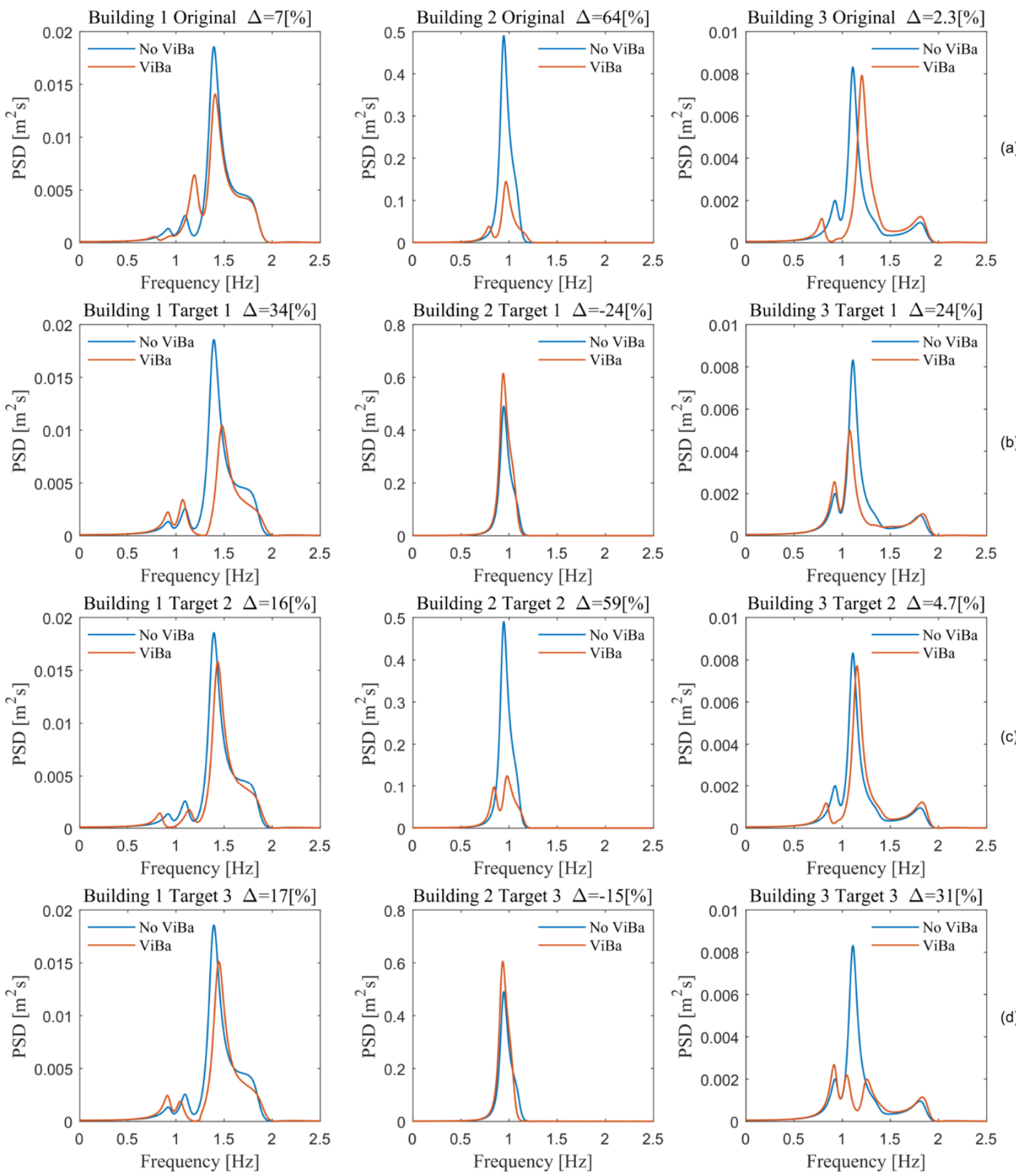

Figure 3 Comparison of the relative respose power spectral densities for the three strucutre with and without the ViBa: a) design case with no scaling factors b) target structure 1, c) target structure 2 and d) target structure 3

unprotected system. Similar results are depicted in Figure 3c where structure 2 is considered as a target. Significant reduction is achieved in the central structure while no detrimental effects are observed in the additional structures. Finally, the case of structure 3 as main target of the design are depicted in Figure 3d a significant reduction is achieved in building three, moderate improvement in structure one and the effects are detrimental in structure two. Figure 4 shows an additional optimization scenario in which the scaling factors are calibrated in such a way that the relative 
response statistical moments possess the same weight. Interestingly, the optimal solution still converge toward the central structure with also an evident, although minor reduction in the nearby structures.
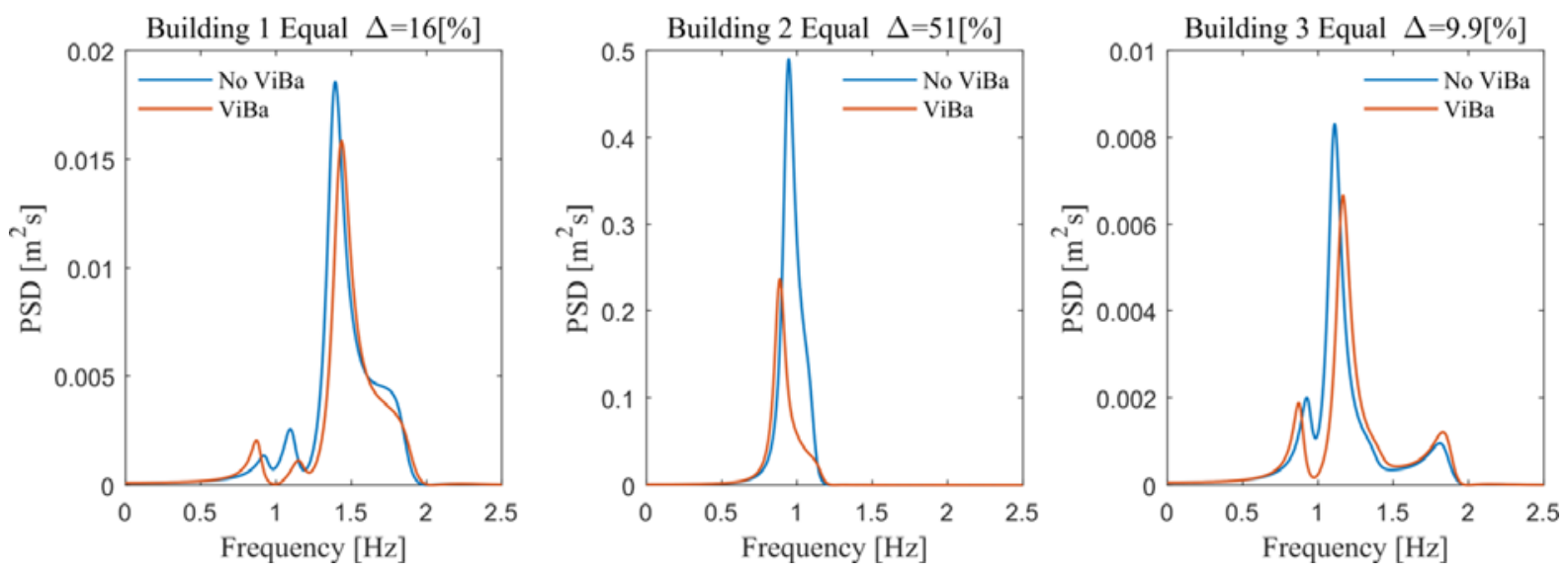

Figure 4 Comparison of the relative respose power spectral densities for the three structures with and without the $\mathrm{ViBa}$

Table 6 Optimal stiffness of the ViBa device for all the cases considered

\begin{tabular}{lll}
\hline Case & Units/Parameter & $k_{v}$ \\
\hline Original & $\mathrm{N} / \mathrm{m}$ & $3.24 \mathrm{E} 7$ \\
\hline Target 1 & $\mathrm{~N} / \mathrm{m}$ & $5.67 \mathrm{E} 8$ \\
\hline Target 2 & $\mathrm{~N} / \mathrm{m}$ & $3.67 \mathrm{E} 7$ \\
\hline Target 3 & $\mathrm{~N} / \mathrm{m}$ & $1.44 \mathrm{E} 8$ \\
\hline All & $\mathrm{N} / \mathrm{m}$ & $4.44 \mathrm{E} 7$ \\
\hline
\end{tabular}

Finally, a Monte Carlo Simulation with 100 samples of stationary Gaussian white noise is performed. Each signal is sampled at $100 \mathrm{~Hz}$ and modulated by using the modulating function $f(t)$ defined by Jennings et al. (1969) as follows;

$$
f(t)=\left\{\begin{array}{cc}
\left(\frac{t}{t_{1}}\right)^{2} & t<t_{1} \\
1 & t_{1} \leq t \leq t_{2} \\
e^{-\beta\left(t-t_{2}\right)} & t>t_{2}
\end{array}\right.
$$

with $\beta=\frac{9}{T_{s}}, t_{1}=\frac{1.5}{\beta}, t_{2}=\frac{10.5}{\beta}$. The total time length of the realization is 20 s with stationary part equals to $T_{s}=15 \mathrm{~s}$. 
Table 7 Percentage reduction of the average peak relative displacement through MCS

\begin{tabular}{llll}
\hline & Structure 1 & Structure 2 & Structure 3 \\
\hline \multirow{2}{*}{ Reduction } & $7.2 \%$ & $28.0 \%$ & $3.87 \%$ \\
\hline
\end{tabular}

The two scenarios determined with the same parameters used to generate the PSDs in Figure 4, are analysed. The results, in terms of reduction of the average peak relative displacement, between system with and without ViBa, are reported in Table 7. It is worth noting that the results achieved by MCS are in agreement with the results expressed in reduction of area reported in Figure 4.

\section{Concluding remarks}

The Vibrating Barrier ( $\mathrm{ViBa}$ ) is a non-localized passive seismic device hosted in the soil and detached from the structures, able to mitigate the dynamic response of one or more buildings. In this paper, the design of the $\mathrm{ViBa}$ to protect buildings in urban environment undergoing stochastic ground motion excitation has been accomplished. Buildings have been modelled as 2-DOF systems coupled together through linear elastic springs representing soil structure and structure soil structure interaction mechanism whereas the ViBa device has been designed as a vibrating structure embedded in a foundation buried in the soil. The proposed design principle is based on the minimization of a penalty function encompassing the stationary second order statistics related to the peak response. Numerical analyses have been carried out for a scenario of three buildings assumed to be extracted from a urban environment. The proposed design has been used to obtain the optimal values of the ViBa in reducing the relative second order response statistical moments. The use of scaling factors to shift the solution toward a given structure within the cluster has been also explored. Results have shown that the ViBa is able to protect a cluster of buildings with a significant reduction of the seismic energy up to 59\%. Finally, a Monte Carlo Simulation has been performed with 100 samples of stationary Gaussian white noise excitations. A relevant $28 \%$ of reduction of the average peak displacement has been achieved by using the proposed design method to calibrate the ViBa. Due to the different frequency characteristics of the structures under consideration higher level of protection can be achieved by the ViBa device hosting additional vibrating masses so to cover a larger frequency spectrum.

\section{References}

Aldaikh, H., Alexander, N.A., Ibraim, E., Oddbjornsson, O., 2015. Two dimensional numerical and experimental models for the study of structure-soil-structure interaction involving three buildings. Computers \& Structures 150, 79-91.

Aldaikh, H., Alexander, N.A., Ibraim, E., Knappett, J., 2016. Shake table testing of the dynamic interaction between two and three adjacent buildings (SSSI). Soil Dynamics and Earthquake Engineering 89, 219-232.

Alexander, N.A., Ibraim, E., Aldaikh, H., 2013. A simple discrete model for interaction of adjacent buildings during earthquakes. Computers \& Structures 124, 1-10.

Cacciola, P., Colajanni, P., Muscolino, G., 2005. A modal approach for the evaluation of the response sensitivity of structural systems subjected to non-stationary random processes. Computer Methods in Applied Mechanics and Engineering 194, 4344-4361.

Cacciola, P., Maugeri, N. and Muscolino, G. 2011. Structural identification through the measure of deterministic and stochastic time-domain dynamic response. Computers \& Structures 89, 1812-1819.

Cacciola, P., Espinosa, M.G., Tombari, A., 2015. Vibration control of piled-structures through structure-soil-structure-interaction. Soil Dynamics and Earthquake Engineering 77, 47-57. 
Cacciola, P., Tombari, A., 2015. Vibrating barrier: a novel device for the passive control of structures under ground motion. Proceedings of the Royal Society of London A: Mathematical, Physical and Engineering Sciences 471.

Colombi, A., Colquitt, D., Roux, P., Guenneau, S., Craster, R.V., 2016. A seismic metamaterial: The resonant metawedge. Scientific Reports 6, 27717.

Friswell, M.I., Mottershead, J.E., 1995. Finite Element Model Updating in Structural Dynamics, Solid Mechanics and its Applications. Springer Netherlands, Dordrecht.

Guéguen, P., Bard, P.-Y., Chavez-Garcia, F., 2002. Site-City Interaction in Mexico City-Like environments: An Analytical Study. Bulletin of the Seismological Society of America 92, 794-811.

Kham, M., Semblat, J.-F., Bard, P.-Y., Dangla, P., 2006. Seismic Site-City Interaction: Main Governing Phenomena through Simplified Numerical Models. Bulletin of the Seismological Society of America 96, 1934-1951.

Kobori, T., Minai, Ryoichiro, Kusakabe, Kaoru, 1973. Dynamical Characteristics of Soil-Structure Cross-Interaction System, I. Bulletin of the Disaster Prevention Research Institute 22, 111-151.

Mulliken, J.S., Karabalis, D.L., 1998. Discrete model for dynamic through-the-soil coupling of 3-D foundations and structures. Earthquake Engineering \& Structural Dynamics 27, 687-710.

Schwan, L., Boutin, C., Padrón, L.A., Dietz, M.S., Bard, P.-Y., Taylor, C., 2016. Site-city interaction: theoretical, numerical and experimental crossed-analysis. Geophysical Journal International 205, 10061031.

Semblat, J.-F., Kham, M., Bard, P.-Y., 2008. Seismic-Wave Propagation in Alluvial Basins and Influence of Site-City Interaction. Bulletin of the Seismological Society of America 98, 2665-2678.

Tombari, A., Zentner, I., Cacciola, P., 2016. Sensitivity of the stochastic response of structures coupled with vibrating barriers. Probabilistic Engineering Mechanics 44, 183-193.

Tombari, A., Garcia Espinosa, M., Alexander, N.A., Cacciola, P., 2018. Vibration control of a cluster of buildings through the Vibrating Barrier. Mechanical Systems and Signal Processing 101, 219-236.

Woods, R.D., 1968. Screening of surface waves in soils. J. Soil Mech. Found. Eng. Div., ASCE 94, 951-979. 